\title{
Epigallocatechin-3-gallate inhibits nicotine-induced migration and invasion by the suppression of angiogenesis and epithelial-mesenchymal transition in non-small cell lung cancer cells
}

\author{
JINGLI SHI ${ }^{1,2^{*}}$, FEI LIU ${ }^{1,2^{*}}$, WENZHANG ZHANG ${ }^{1,2}$, XIN LIU $^{1,2}$, BIHUA LIN $^{1,2}$ and XUDONG TANG ${ }^{1,2}$ \\ ${ }^{1}$ Institute of Biochemistry and Molecular Biology, and ${ }^{2}$ Guangdong Provincial Key Laboratory of \\ Medical Molecular Diagnostics, Guangdong Medical College, Zhanjiang, Guangdong 524023, P.R. China
}

Received December 3, 2014; Accepted March 12, 2015

DOI: 10.3892/or.2015.3889

\begin{abstract}
Epigallocatechin-3-gallate (EGCG), the most abundant polyphenol in green tea extract, has been found to have anticancer activities in various types of cancer. However, the underlying mechanisms are not completely clear. In the present study, the effects of EGCG on migration, invasion, angiogenesis and epithelial-mesenchymal transition (EMT) induced by nicotine in A549 non-small cell lung cancer (NSCLC) cells were investigated, and the underlying molecular mechanisms were preliminarily examined. The results showed that different concentrations of EGCG significantly inhibited nicotine-induced migration and invasion. Moreover, EGCG reversed the upregulation of $\mathrm{HIF}-1 \alpha$, vascular endothelial growth factor (VEGF), COX-2, p-Akt, p-ERK and vimentin protein levels and the downregulation of $\mathrm{p} 53$ and $\beta$-catenin protein levels mediated by nicotine in A549 cells, but had no significant effect on their mRNA levels. Furthermore, EGCG markedly inhibited HIF-1 $\alpha$-dependent angiogenesis induced by nicotine in vitro and in vivo, and suppressed HIF-1 $\alpha$ and VEGF protein expression induced by nicotine in A549 xenografts of nude mice. Taken together, the results indicated that EGCG inhibited nicotine-induced angiogenesis and EMT, leading to migration and invasion in A549 cells. The results of the present study suggested that
\end{abstract}

Correspondence to: Professor Xudong Tang, Institute of Biochemistry and Molecular Biology, Guangdong Medical College, 2 Wenming Donglu, Xiashan, Zhanjiang, Guangdong 524023, P.R. China

E-mail: tangxudong2599@126.com

*Contributed equally

Abbreviations: EGCG, epigallocatechin-3-gallate; NSCLC, nonsmall cell lung cancer; EMT, epithelial-mesenchymal transition

Key words: epigallocatechin-3-gallate, nicotine, non-small cell lung cancer, angiogenesis, epithelial-mesenchymal transition, migration, invasion
EGCG can be developed into a potential agent for the prevention and treatment of smoking-associated NSCLC.

\section{Introduction}

Lung cancer is a leading cause of cancer-related mortality worldwide. Non-small cell lung cancer (NSCLC) comprises $\sim 80 \%$ of all lung cancers (1). Cigarette smoking is the most important risk factor of NSCLC. Nicotine, the principal chemical component in tobacco smoke associated with addiction, is involved in the progression and metastasis of NSCLC (2). Accumulating experimental evidence has demonstrated that nicotine and its metabolites promotes migration and invasion in lung cancer cells (3-5). Angiogenesis, the formation of new blood vessels from a pre-existing vascular network, is essential for tumor migration, invasion and metastasis (6). Epithelialmesenchymal transition (EMT), a process by which epithelial cells lose the epithelial phenotype and gain the mesenchymal phenotype, is largely thought to play a key role in invasion and metastasis (7). Nicotine has also been found to enhance angiogenesis $(8,9)$ and EMT $(4,5)$ in NSCLC cells. Angiogenesis and EMT play a crucial role in the development and progression of NSCLC $(6,10)$. Thus the inhibitors of angiogenesis and EMT mediated by nicotine may be developed into chemopreventive agents of smoking-associated NSCLC.

Green tea, widely consumed in Asia, contains polyphenolic compounds that account for $30 \%$ of the dry weight of the leaves. Previous epidemiological studies and meta-analysis have revealed that green tea consumption reduces the risk of lung cancer $(11,12)$. The chemopreventive effects of green tea are mediated by polyphenolic compounds known as catechins including (-)-epigallocatechin-3-gallate (EGCG), (-)-epicatechin-3-gallate (ECG), (-)-epigallocatechin (EGC) and (-)-epicatechin (EC), with EGCG being the most abundant and powerful catechin in cancer prevention and treatment $(13,14)$. A growing body of evidence has demonstrated that EGCG inhibits migration and invasion in a variety of cancer cells (15-17). In a previous study, we found that EGCG inhibited angiogenesis by downregulating hypoxia- and serum-induced hypoxia-inducible factor $1 \alpha(\mathrm{HIF}-1 \alpha)$ protein accumulation and vascular endothelial growth factor (VEGF) expression in 
human cervical carcinoma and hepatoma cells (18). Previously, we demonstrated that EGCG inhibits human papillomavirus (HPV)-16 oncoprotein- and IGF-1-induced angiogenesis in NSCLC by targeting HIF-1 $\alpha(19,20)$. Furthermore, nicotine induces HIF-1 $\alpha$ protein expression in NSCLC cells through the nicotinic acetylcholine receptor-mediated signaling pathway (8). However, the effect of EGCG on angiogenesis induced by nicotine in NSCLC cells has not been reported.

EMT promotes the invasion and metastasis of various types of cancers including NSCLC $(7,10)$. EGCG has been found to inhibit EMT mediated by a variety of factors (21-23). Liu et al have reported that EGCG inhibited transforming growth factor- $\beta$ (TGF- $\beta$ )-mediated EMT in NSCLC cells via inhibition of the Smad 2 and Erk1/2 signaling pathways (21). Ko et al also found that EGCG negatively affected TGF- $\beta 1$-mediated EMT by suppressing the acetylation of Smad2 and Smad3 in A549 NSCLC cells (22). However, the effect of EGCG on nicotineinduced EMT in NSCLC cells remains to be determined.

In the present study, we determined the effect of EGCG on nicotine-induced migration, invasion, angiogenesis and EMT in NSCLC cells. To the best of our knowledge, the results of the present study showed for the first time that, EGCG inhibited nicotine-induced angiogenesis and EMT in A549 NSCLC cells, leading to the suppression of migration and invasion. Furthermore, we found that EGCG suppressed Akt and ERK1/2 activation induced by nicotine. These findings provide further evidence with regard to the underlying molecular mechanisms involved in the anticancer effect of EGCG on smoking-associated NSCLC.

\section{Materials and methods}

Drug and reagents. EGCG was purchased from Sigma (St. Louis, MO, USA), dissolved in distilled water at a concentration of $100 \mathrm{mM}$ and stored at $-80^{\circ} \mathrm{C}$ as a stock solution. Nicotine was also obtained from Sigma. Complete protease inhibitor cocktail was from Roche (Mannheim, Germany). Mouse anti-human HIF-1 $\alpha$ monoclonal antibody was purchased from BD Transduction Laboratories (San Diego, CA, USA). Vimentin, $\beta$-catenin, total and phosphorylated Akt (Ser473) or ERK1/2 (Thr202/Tyr204) antibodies were purchased from Cell Signaling Technology (Beverly, MA, USA). Antibody for $\beta$-actin was purchased from Beyotime Biotechnology Corporation (Shanghai, China). Horseradish peroxidase (HRP)-conjugated secondary antibodies were obtained from Cell Signaling Technology. The human VEGF ELISA Development kit was purchased from Wuhan Boster Bio-Engineering Co., Ltd. (Wuhan, China). One-Step SYBR ${ }^{\circledR}$ PrimeScript $^{\circledR}$ RT-qPCR (no. DRR086A) was purchased from Takara Biotechnology Co., Ltd. (Dalian, China). Transfection reagent (Lipofectamine ${ }^{\mathrm{TM}}$ 2000) was obtained from Invitrogen Corporation (Carlsbad, CA, USA). An In Vitro Angiogenesis Assay kit (ECM625) was obtained from Millipore (Temecula, CA, USA). An In Vivo Angiogenesis Assay kit (no. 354248) was obtained from BD Biosciences (Bedford, MA, USA). HiCN Hemoglobin Detection kit was purchased from Shanghai Rongsheng Biotech Co., Ltd. (Shanghai, China).

Cell culture and treatment with EGCG. The human A549 NSCLC cell line (adenocarcinoma cell line) and human umbil- ical vein endothelial cells (HUVECs) were obtained from the American Type Culture Collection (ATCC; Rockville, MD, USA). The cells were cultured in RPMI-1640 medium supplemented with $10 \%$ fetal bovine serum (FBS), penicillin $(100 \mathrm{U} / \mathrm{ml})$ and streptomycin $(100 \mu \mathrm{g} / \mathrm{ml})$. Cultures were maintained at $37^{\circ} \mathrm{C}$ in a humidified atmosphere with $5 \% \mathrm{CO}_{2}$. Exponentially growing cells ( $~ 80 \%$ confluence) in complete media were pretreated for $1 \mathrm{~h}$ with different concentrations of EGCG, followed by exposure to nicotine $(5 \mu \mathrm{M})$ for the indicated time points according to the purpose of the experiment.

Wound-healing assays. A549 cells were plated and grown to $80 \%$ confluence in a $24-w e l l$ plate (Corning Inc.). The cells were starved in $1 \%$ FBS for $24 \mathrm{~h}$ and then washed with phosphate-buffered saline (PBS). The cells were scratched with a sterile $200 \mu \mathrm{l}$ pipette tip in each well and medium containing $10 \% \mathrm{FBS}$ and $5 \mu \mathrm{M}$ nicotine in the presence or absence of EGCG. After 24 and $48 \mathrm{~h}$, the wounds were observed and images were captured. The data were representative of three independent experiments.

Cell invasion assays. The invasive ability of A549 cells was assayed using 24-well invasion chambers (Corning Inc.) with filters coated with Matrigel (BD Biosciences) on the upper surface with $8.0-\mu \mathrm{m}$ pores. Briefly, Matrigel was applied to the upper surface of the chambers. A549 cells $\left(5 \times 10^{4}\right)$ in $0.2 \mathrm{ml}$ medium were added into the upper chamber, and $0.5 \mathrm{ml} 10 \%$ FBS medium in the presence or absence of $5 \mu \mathrm{M}$ nicotine or different concentrations of EGCG $(10,25,50$ and $100 \mu \mathrm{M})$ was added into the lower chamber. After incubation at $37^{\circ} \mathrm{C}$ with $5 \%$ $\mathrm{CO}_{2}$ for $36 \mathrm{~h}$, non-migrating A549 cells on the upper surface of the chambers were removed by wiping with cotton swabs. The filters were stained with hematoxylin for $30 \mathrm{~min}$. The number of cells migrating on the other side of the filters was counted. The data are representative of three independent experiments.

Western blot analysis. Nicotine- or EGCG-treated A549 cells were lysed with cell lysis buffer containing $20 \mathrm{mM}$ Tris (pH 7.5), $150 \mathrm{mM} \mathrm{NaCl}, 1 \%$ Triton $\mathrm{X}-100$ and sodium pyrophosphate, $\beta$-glycerophosphate, EDTA, $\mathrm{Na}_{3} \mathrm{VO}_{4}$, leupeptin phenylmethyl-sulfonyl fluoride (PMSF), and complete protease inhibitor cocktail (Roche) followed by incubation at $4^{\circ} \mathrm{C}$ for $1 \mathrm{~h}$. The lysates were ultra-sonicated and centrifuged at $12,000 \mathrm{x} \mathrm{g}$ for $10 \mathrm{~min}$. Protein concentrations were determined by BCA methods. Protein $(100 \mu \mathrm{g})$ was separated on $10 \%$ polyacrylamide-SDS gel and electroblotted onto PVDF membranes (Millipore). After blocking with 5\% skim milk, the membrane was incubated overnight at $4^{\circ} \mathrm{C}$ with primary antibody against HIF- $1 \alpha$, vimentin, $\beta$-catenin, total-Akt (t-Akt), phosphorylated Akt (p-Akt), total-ERK1/2 (t-ERK1/2), or phosphorylated-ERK1/2 (p-ERK1/2), followed by incubation with HRP-conjugated secondary antibodies $(1: 1,000)$. As a loading control, the blots were stripped and re-probed with anti- $\beta$-actin antibody.

$R T-q P C R$ analysis. Total RNA was isolated from cells using TRIzol ${ }^{\circledR}$ reagent (Invitrogen). RT-qPCR analysis of HIF-1 $\alpha$ and VEGF mRNA levels was performed using One-Step SYBR $^{\circledR}$ PrimeScript ${ }^{\circledR}$ RT-PCR (Biotechnology Co., Ltd.) according to the manufacturer's instructions. The primers 
designed for RT-qPCR were as follows: HIF- $1 \alpha$, forward: 5'-TCTGGGTTGAAACTCAAGCAACTG-3' and reverse: 5'-CAACCGGTTTAAGGACACATTCTG-3' (GenBank, NM_001243084.1); human VEGF, forward: 5'-TGCTTCTGA GTTGCCCAGGA-3' and reverse: 5'-TGGTTTCAATGGTG TGAGGACATAG-3' (GenBank, NM_003376.5); p53, forward: 5'-GAGGCCTTGGAACTCAAGGATG-3' and reverse: 5'-TCAGGCCCTTCTGTCTTGAACA-3' (GenBank, NM_000546.4); COX-2, forward: 5'-CTGTAACCAAGATGG ATGCAA-AGA-3' and reverse: 5'-GTCAGTGACAATGAGA TGTGGAA-3' (GenBank, NM_000963.2); $\beta$-actin, forward: 5'-TGGCACCCAGCACAATGAA-3' and reverse: 5'-CTAAGT CATAGTCCGCCTAGAAGCA-3' (GenBank, NM_001101.3). The primers were produced by Takara Biotechnology Co., Ltd. The thermocycling conditions used were: $42^{\circ} \mathrm{C}$ for $5 \mathrm{~min}, 95^{\circ} \mathrm{C}$ for $10 \mathrm{sec}$, followed by $40 \mathrm{cycles}$ at $95^{\circ} \mathrm{C}$ for $5 \mathrm{sec}$, and $60^{\circ} \mathrm{C}$ for $31 \mathrm{sec}$. The size of the PCR product of HIF-1 $\alpha$, VEGF, p53, COX-2 and $\beta$-actin was 150, 176, 137, 195 and $186 \mathrm{bp}$, respectively. The relative HIF-1 $\alpha$, VEGF, p53 and COX-2 mRNA levels were normalized to $\beta$-actin. The experiment was repeated in triplicate.

Enzyme-linked immunosorbent assay (ELISA). The concentration of VEGF protein in the conditioned media derived from nicotine- or EGCG-treated A549 cells was determined using a human VEGF ELISA development kit according to the manufacturer's instructions. The results were normalized to the cell number $\left(5 \times 10^{3}\right.$ cells). The experiments were repeated in triplicate.

RNA interference. HIF- $1 \alpha$-siRNA (sense strand, 5'-GCCGCUC AAUUUAUGAAUATT-3') and non-specific (NS)-siRNA (sense strand, 5'-UUCUCCGAACGUGUCACGUTT-3') duplexes were generated by Shanghai GenePharma Co., Ltd. (Shanghai, China). Transfection was performed using the Lipofectamine $^{\mathrm{TM}} 2000$ transfection reagent according to the manufacturer's instructions.

In vitro angiogenesis assay. An in vitro angiogenesis assay kit was employed according to the manufacturer's instructions (Millipore). HUVECs $\left(5 \times 10^{3}\right.$ cells/well) were seeded onto the surface of 96-well cell culture plates pre-coated with polymerized ECMatrix ${ }^{\mathrm{TM}}$ and then incubated at $37^{\circ} \mathrm{C}$ for $6-8 \mathrm{~h}$ in the conditioned media derived from nicotine-treated A549 cells transfection with HIF-1 $\alpha$-siRNA or NS-siRNA. In parallel studies, HUVECs were cultured in the conditioned media derived from nicotine-treated A549 cells pretreated with $100 \mu \mathrm{M}$ of EGCG. Tubule formation was observed under a phase-contrast microscope. The total tube length in three random view-fields/wells was measured by Scion Image software and the average value was calculated. The experiment was repeated in triplicate.

In vivo angiogenesis assay. Male nude mice (BALB/C nude) (6-8 weeks old) were purchased from the Animal Center of Southern Medical University (Guangzhou, China). Experiments with animals were undertaken in accordance with the institution guidelines of the Institutional Animal Care and Use Committee of Southern Medical University and Guangdong Medical College. Nicotine- or EGCG-treated
A549 cells were re-suspended in serum-free media at a density of $8.0 \times 10^{6}$ cells $/ \mathrm{ml}$ and $0.25 \mathrm{ml}\left(2.0 \times 10^{6}\right.$ cells in total $)$ of cell suspension was mixed with the same volume of BD Matrigel Matrix $(0.25 \mathrm{ml})$. The BD Matrigel mixture was subcutaneously injected into the two flanks of nude mice. On day 11, the mice were euthanized and Matrigel plugs were harvested, with half of each Matrigel plug fixed with $10 \%$ neutral formalin for immunohistochemical studies and the other half weighed for the determination of hemoglobin content as previously described (20).

Immunohistochemistry. The expression of HIF-1 $\alpha$ and VEGF proteins in Matrigel plugs was analyzed by immunohistochemistry as previously described (20). Briefly, formalin-fixed Matrigel plugs were paraffin-embedded and sectioned at $4 \mu \mathrm{m}$. The sections were then deparaffinized in xylene, rehydrated through serial dilutions of alcohol and washed in PBS (pH 7.2). The sections were heated in a microwave oven twice for $5 \mathrm{~min}$ in citrate buffer ( $\mathrm{pH}$ 6.0) and then incubated overnight at $4^{\circ} \mathrm{C}$ with primary antibody, followed by incubation with streptavidin/HRP-conjugated secondary antibodies. The conventional streptavidin/peroxidase method (Histostain ${ }^{\mathrm{TM}}$-Plus kits, SP0023; Beijing Biosynthesis Biotechnology, Co., Ltd., Beijing, China) was used to develop signals, and the cells were counterstained with hematoxylin. The sections incubated with secondary antibodies in the absence of primary antibodies served as negative controls.

Statistical analysis. Data are presented as the mean \pm SD for three separate experiments. One-way ANOVA, Bonferroni and Dunnett-T3 were employed for statistical analysis using SPSS 19.0 for windows software. $\mathrm{P}<0.05$ was considered to indicate a statistically significant result.

\section{Results}

EGCG inhibits nicotine-induced migration and invasion in A549 cells. A wound-healing assay was carried out to assess whether EGCG affects the migratory properties of A549 cells induced by nicotine. Similar to recent results (3), we found that nicotine enhanced A549 cell migration (Fig. 1A). However, EGCG significantly inhibited the migration of A549 cells induced by nicotine in a dose-dependent manner, with the maximal efficiency being observed at $100 \mu \mathrm{M} \mathrm{EGCG}$ treatment (Fig. 1A). The effect of EGCG on invasion induced by nicotine in A549 cells was examined. As shown in Fig. 1B and C, treatment of cells with nicotine promoted cell invasive property. However, EGCG inhibited the invasive property of A549 cells induced by nicotine in a dose-dependent manner, and the maximum effect was observed at $100 \mu \mathrm{M}$ EGCG treatment. Taken together, the results indicated that EGCG inhibited nicotine-induced migration and invasion in A549 cells.

EGCG reverses the upregulation of HIF-1 $\alpha, V E G F, C O X-2$, $p$-Akt, $p$-ERK and vimentin protein levels and the downregulation of $p 53$ and $\beta$-catenin protein levels mediated by nicotine in A549 cells. To determine the effects of EGCG on angiogenesis and EMT mediated by nicotine in A549 cells, the expression of angiogenesis- and EMT-associated proteins was analyzed. Western blot analysis was carried out to assess 
A

\section{Nicotine $(5 \mu \mathrm{M})$ EGCG $(\mu \mathrm{M})$}

$\mathbf{0 ~ h}$

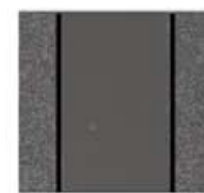

24 h

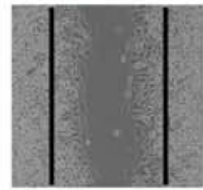

$48 \mathrm{~h}$

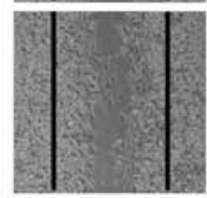

B

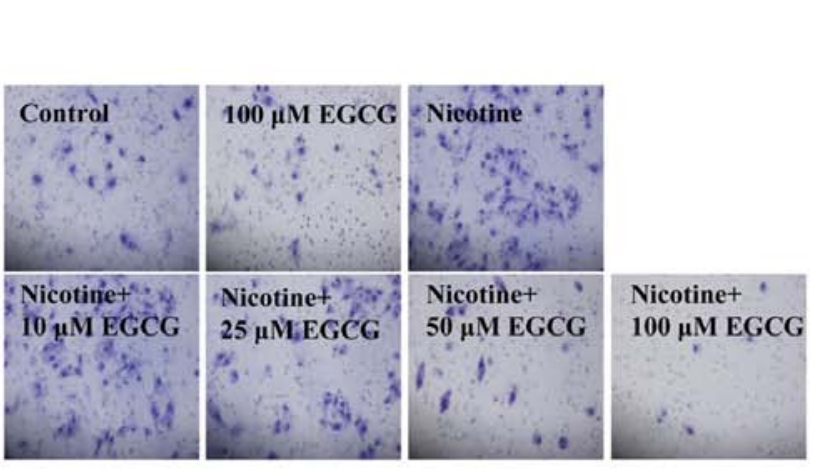

100
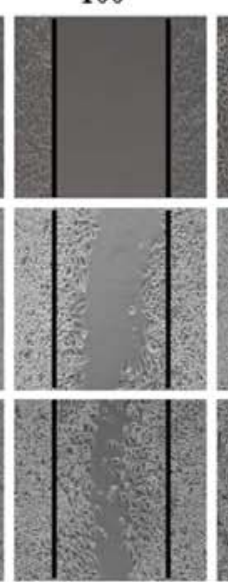

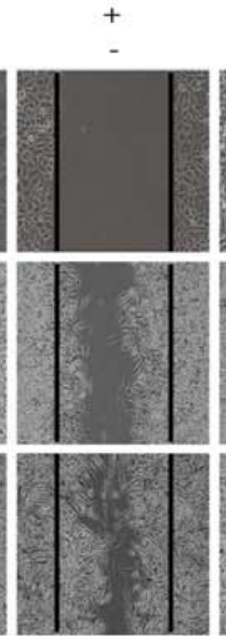

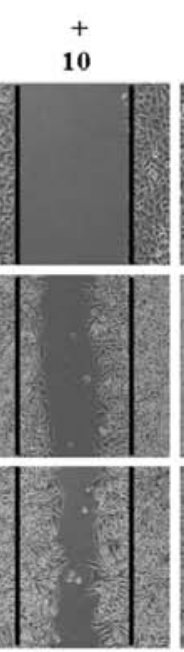

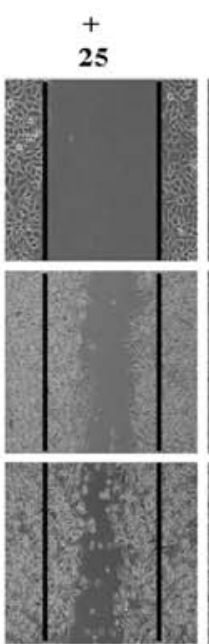

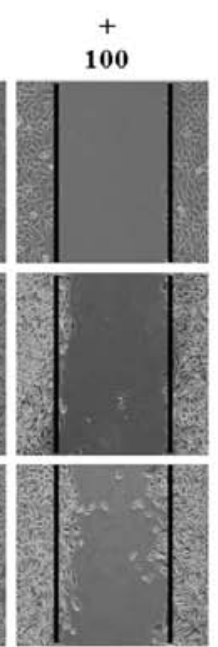

C

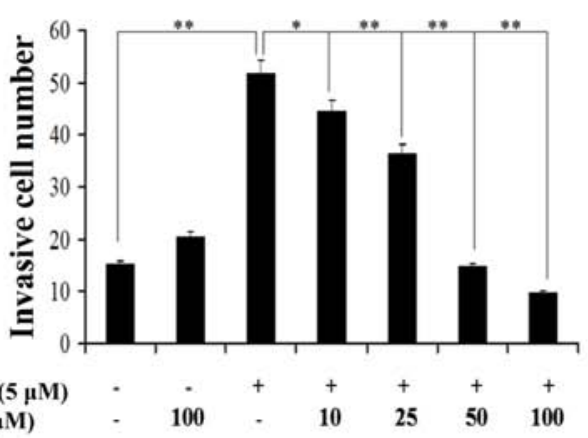

Figure 1. EGCG inhibits nicotine-induced migration and invasion in A549 NSCLC cells. (A) The migration of A549 cells exposed to nicotine in the presence or absence of EGCG was analyzed by a wound-healing assay. (B and C) The invasion of A549 cells exposed to nicotine in the presence or absence of EGCG was detected by the Matrigel invasion chamber. (B) Images are representative of results from three independent experiments. (C) The number of invasive cells from (B). The data are presented as the mean \pm SD from three replicate experiments. ${ }^{*} \mathrm{P}<0.05{ }^{* *} \mathrm{P}<0.01$. EGCG, epigallocatechin-3-gallate; NSCLC, non-small cell lung cancer.

the expression of HIF-1 $\alpha$, p53, COX-2, vimentin, $\beta$-catenin, p-Akt and p-ERK, and ELISA was performed to determine VEGF concentrations in the conditioned media derived from differentially treated cells. The results showed that nicotine significantly upregulated the expression of HIF-1 $\alpha$, VEGF, COX-2 and vimentin proteins in A549 cells, but downregulated the expression of $\beta$-catenin and $\mathrm{p} 53$ proteins in A549 cells (Fig. 2A, B and D). However, EGCG significantly reversed the effects of nicotine on the expression of these proteins in A549 cells (Fig. 2A, B and D). We also assessed whether EGCG affects the activation of Akt and ERK induced by nicotine in A549 cells. The results showed that EGCG inhibited the expression of p-Akt and p-ERK induced by nicotine in A549 cells (Fig. 2C). Taken together, our results suggested that EGCG inhibited angiogenesis and EMT mediated by nicotine in A549 cells.

EGCG has no significant effect on the expression of HIF-1 $\alpha$, $V E G F, C O X-2$ and $p 53$ mRNA mediated by nicotine in A549 cells. Since EGCG affected the expression of HIF-1 $\alpha$, VEGF, p53 and COX-2 proteins induced by nicotine in A549 cells, to assess whether the alteration of HIF- $1 \alpha$, VEGF, p53 and COX-2 expression can be regulated at the transcriptional levels, we measured mRNA levels by RT-qPCR. As shown in Fig. 3, treatment of cells with nicotine upregulated HIF-1 $\alpha$, VEGF and COX-2 mRNA levels and downregulated the p53 mRNA level as compared to the controls, whereas EGCG treatment had no obvious effects on HIF-1 $\alpha$, VEGF, p53 and COX-2 mRNA levels mediated by nicotine. These results suggested that EGCG affected the expression of HIF-1 $\alpha$, VEGF, p53 and COX-2 proteins mediated by nicotine in A549 cells possibly through translational or post-translational mechanisms.

EGCG inhibits HIF-1 $\alpha$-dependent angiogenesis induced by nicotine in vitro and in vivo, and suppressed HIF-1a and VEGF protein expression induced by nicotine in A549 xenografts of nude mice. To determine the effects of EGCG on nicotine-induced angiogenesis in A549 cells, an in vitro angiogenesis model was employed to evaluate the capillary tube formation of HUVECs stimulated by the conditioned media derived from A549-treated cells. Since VEGF is one of the downstream genes of HIF-1 $\alpha(24,25)$, to investigate whether the increased expression of VEGF and angiogenesis induced by nicotine in A549 cells was mediated by HIF-1 $\alpha$, A549 cells were transfected with HIF-1 $\alpha$-specific siRNA (HIF-1 $\alpha$-siRNA) or HIF-1 $\alpha$ non-specific siRNA (NS-siRNA). The results showed that A549 cells treated with nicotine enhanced the ability to stimulate the formation of capillary tube-like structures by HUVECs as compared to the controls. Furthermore, A549 cells treated with EGCG or transfection 
A

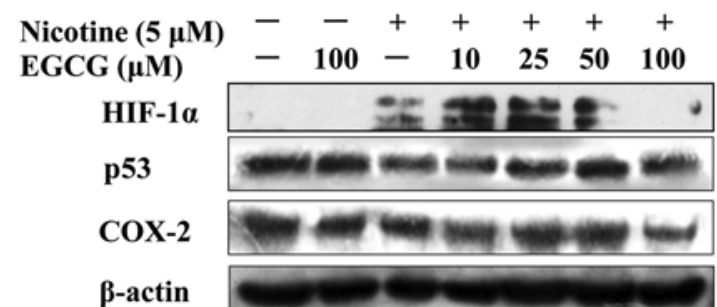

C

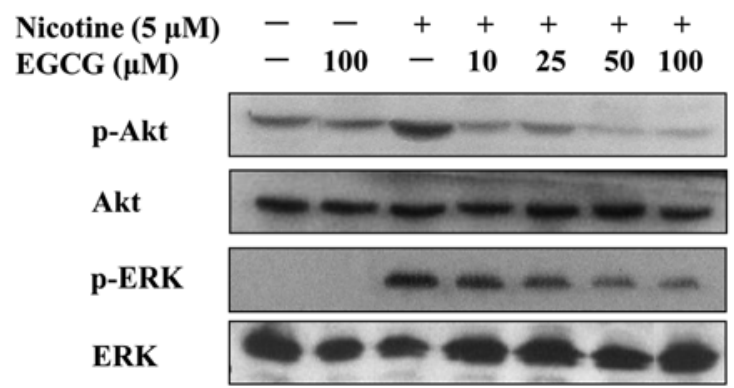

B

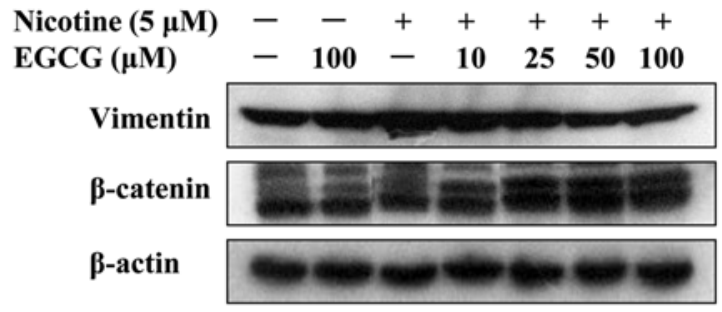

D

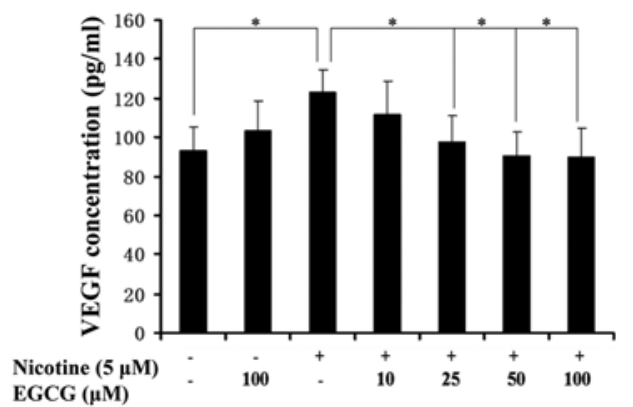

Figure 2. EGCG reverses the upregulation of HIF-1 $\alpha$, VEGF, COX-2, p-Akt, p-ERK and vimentin protein levels and the downregulation of p53 and $\beta$-catenin protein levels mediated by nicotine in A549 cells. Exponentially growing cells in complete media were pretreated for $1 \mathrm{~h}$ with different concentrations of EGCG, followed by exposure to nicotine $(5 \mu \mathrm{M})$ for $16 \mathrm{~h}$. (A-C) Western blot analysis was performed to determine the expression of HIF-1 $\alpha$, p53, COX-2, vimentin, $\beta$-catenin, $\mathrm{p}$-Akt and p-ERK proteins. (D) VEGF concentration in the conditioned media derived from A549-treated cells was determined by ELISA. The data are presented as the mean \pm SD from three replicate experiments. " $\mathrm{P}<0.05$. EGCG, epigallocatechin-3-gallate; VEGF, vascular endothelial growth factor.

A

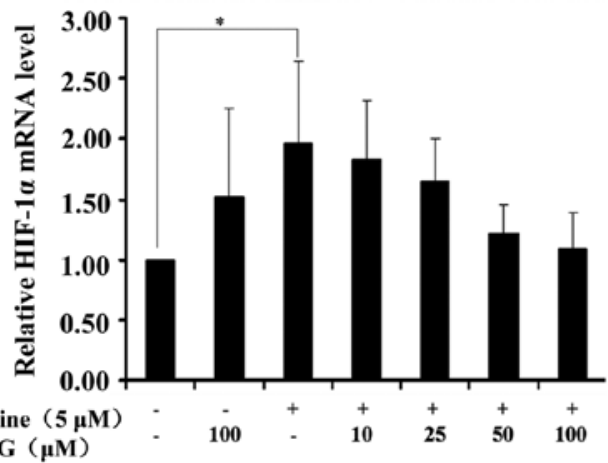

C

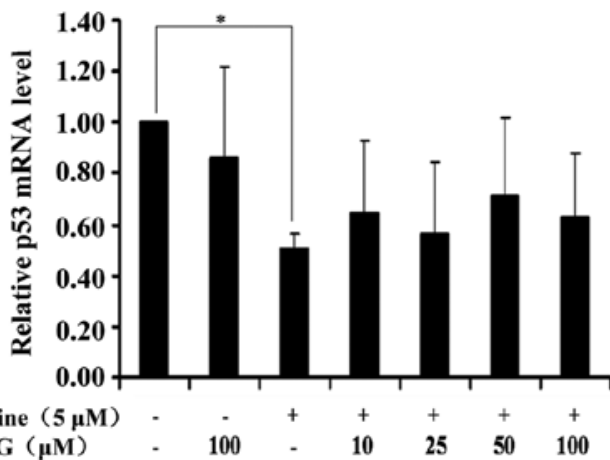

B

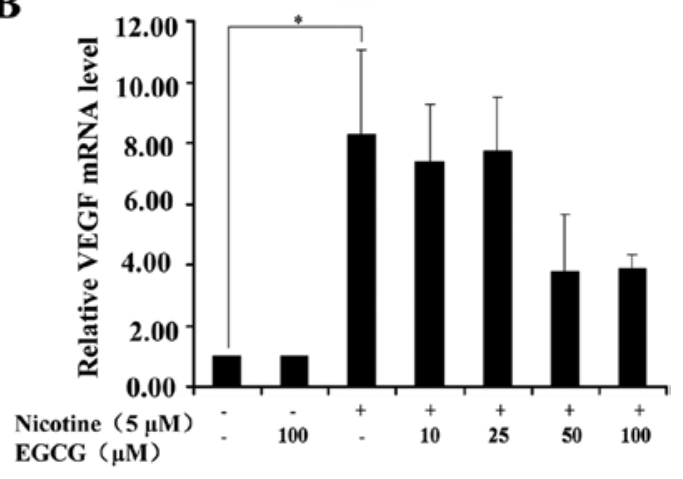

D

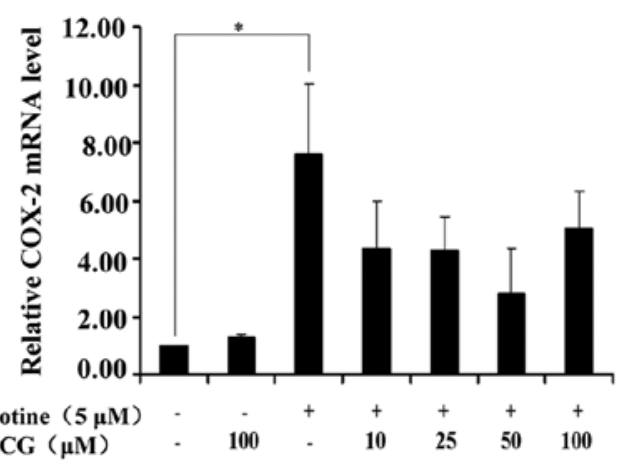

Figure 3. EGCG has no significant effects on the expression of HIF-1 $\alpha$, VEGF, p53 and COX-2 mRNA mediated by nicotine in A549 cells. Exponentially growing cells in complete media were pretreated for $1 \mathrm{~h}$ with different concentrations of EGCG, followed by exposure to nicotine $(5 \mu \mathrm{M})$ for $16 \mathrm{~h}$. The expression levels of (A) HIF-1 $\alpha$, (B) VEGF, (C) p53 and (D) COX-2 mRNA were determined by RT-qPCR. The data are presented as the mean \pm SD from three replicate experiments. $* \mathrm{P}<0.05$. EGCG, epigallocatechin-3-gallate; VEGF, vascular endothelial growth factor.

with HIF-1 $\alpha$-siRNA inhibited the nicotine-induced ability to stimulate the formation of capillary tube-like structures by
HUVECs as compared with nicotine-treated cells (Fig. 4A), which was further confirmed by quantification of the total 
A
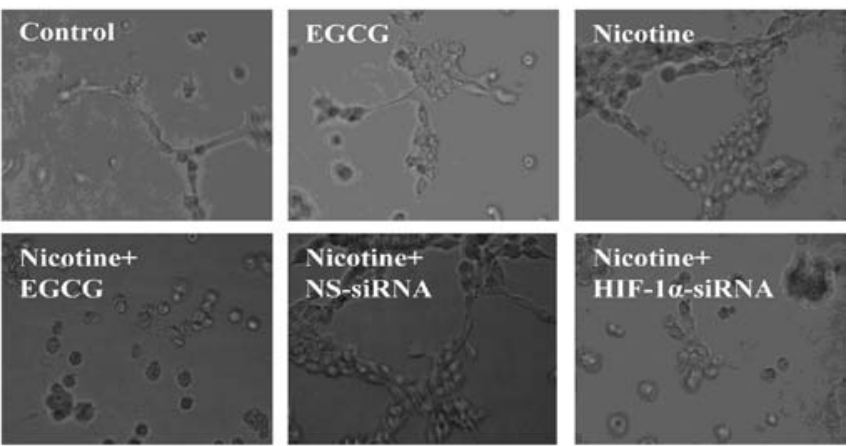

B

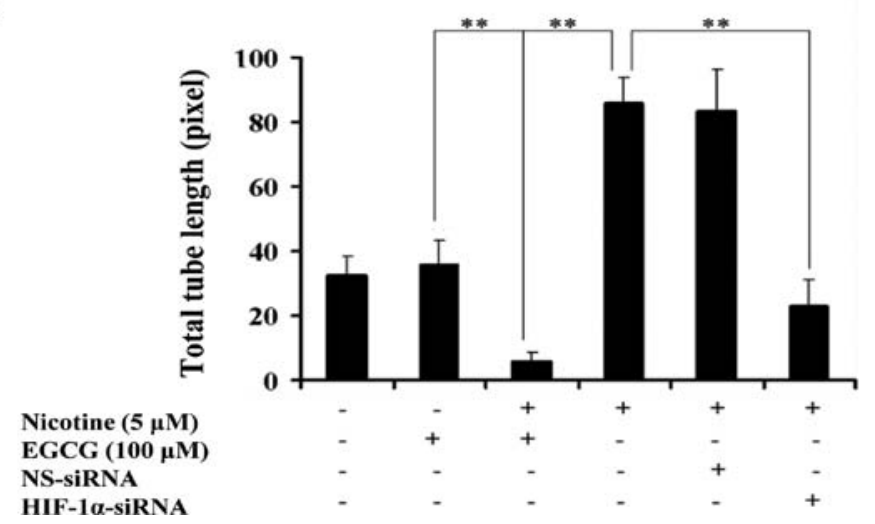

Figure 4. Treatment with EGCG and transfection with HIF-1 $\alpha$-siRNA inhibit nicotine-induced angiogenesis in vitro in A549 cells. (A) Tube formation was observed under a phase-contrast microscope (magnification, x200). The data presented are representative of three separate experiments. (B) The total tube length from (A) in three random view-fields/well was measured by Scion Image software and the average value was calculated. The data are presented as mean \pm SD of three independent experiments. ${ }^{* *} \mathrm{P}<0.01$. EGCG, epigallocatechin-3-gallate.

tube length pixel values $(\mathrm{P}<0.05$; Fig. 4B). However, such inhibition of angiogenic abilities was not observed in NS-siRNA-transfected cells ( $\mathrm{P}>0.05$; Fig. 4A and B).

We performed a Matrigel plug angiogenesis assay using BALB/C nude mice to observe the effect of EGCG on nicotine-induced tumor angiogenesis in vivo. The results showed that Matrigel plugs mixed with nicotine-treated cells obviously induced angiogenesis in vivo (Fig. 5A), showing much higher hemoglobin levels than those in the controls $(\mathrm{P}<0.05$; Fig. 5B). Moreover, Matrigel plugs mixed with EGCG or HIF-1 $\alpha$-siRNA-transfected cells significantly inhibited angiogenesis in vivo stimulated by nicotine (Fig. 5A), showing barely detectable or low hemoglobin levels $(\mathrm{P}<0.05$; Fig. 5B). However, Matrigel plugs mixed with NS-siRNA-transfected cells did not inhibit angiogenesis in vivo induced by nicotine (Fig. 5A and B).

In addition, immunohistochemical results showed that xenografts of A549 cells treated with nicotine exhibited a significantly enhanced expression of HIF-1 $\alpha$ and VEGF proteins as compared to the controls (Fig. 5C). The enhanced expression of HIF-1 $\alpha$ protein was inhibited by transfection with HIF- $1 \alpha-$ siRNA, but not by transfection with NS-siRNA (Fig. 5C). The increase of VEGF expression induced by nicotine was abrogated by transfection with HIF- $1 \alpha$-siRNA, but not by transfection with NS-siRNA. These results indicated that the upregulation of VEGF protein expression induced by nicotine was HIF-1 $\alpha$-dependent in the xenografts of A549 cells. Taken together, these results indicated EGCG markedly inhibited HIF-1 $\alpha$-dependent angiogenesis induced by nicotine in vitro and in vivo, and downregulated HIF-1 $\alpha$ and VEGF protein expression in A549 xenografts of nude mice.

\section{Discussion}

Cancer progression is associated with the abrogation of normal biological characteristics that limit cell proliferation, migration and invasion, eventually leading to metastasis (26-28). Since metastases are a major cause of recurrence and mortality in cancer patients, the development of new drugs that reduce invasion and metastasis is extremely important for the prevention and treatment of cancer. In the present study, we demonstrate that EGCG obviously inhibited the migration and invasion induced by nicotine in A549 cells, suggesting that EGCG holds great potential as a chemopreventive agent in nicotine-associated NSCLC.

EMT is a critical normal process during development and wound healing. However, the properties of EMT were shown to be involved in human pathology, including fibrosis and cancer metastasis $(29,30)$. EMT facilitates tumor cell migration, invasion and metastasis, and therefore may also be a major mechanism of tumor progression (26-28). The results presented in this study showed that nicotine upregulated the expression of vimentin but downregulated the expression level of $\beta$-catenin, an extracellular matrix protein marker. However, treatment with EGCG reversed the expression of vimentin and $\beta$-catenin mediated by nicotine in A549 cells. Previous findings have demonstrated that the decrease in E-cadherin and $\beta$-catenin levels with a concurrent increase in 
A

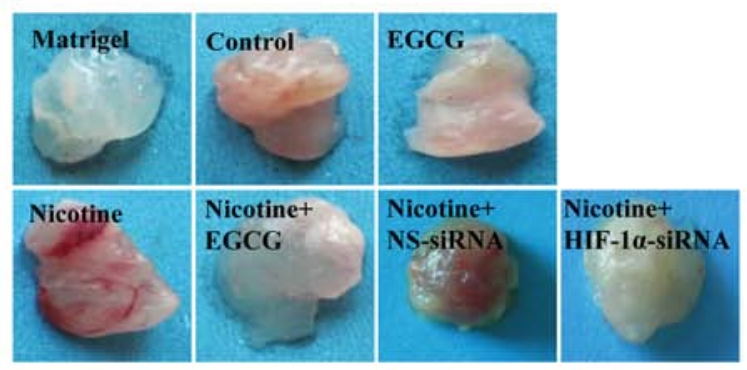

B

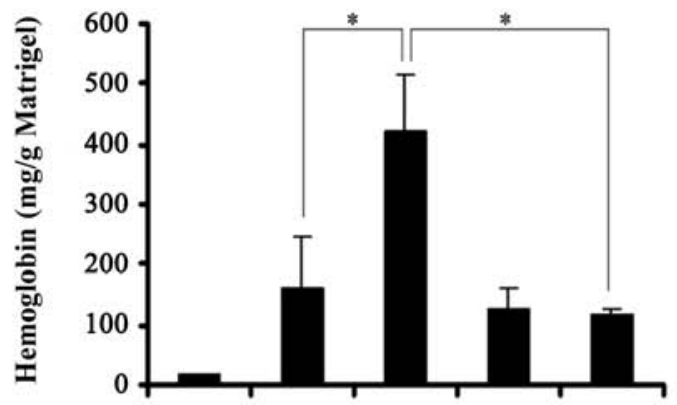

Matrigel

A549

Nicotine $(5 \mu \mathrm{M})$ $\operatorname{EGCG}(\mu \mathrm{M})$
C
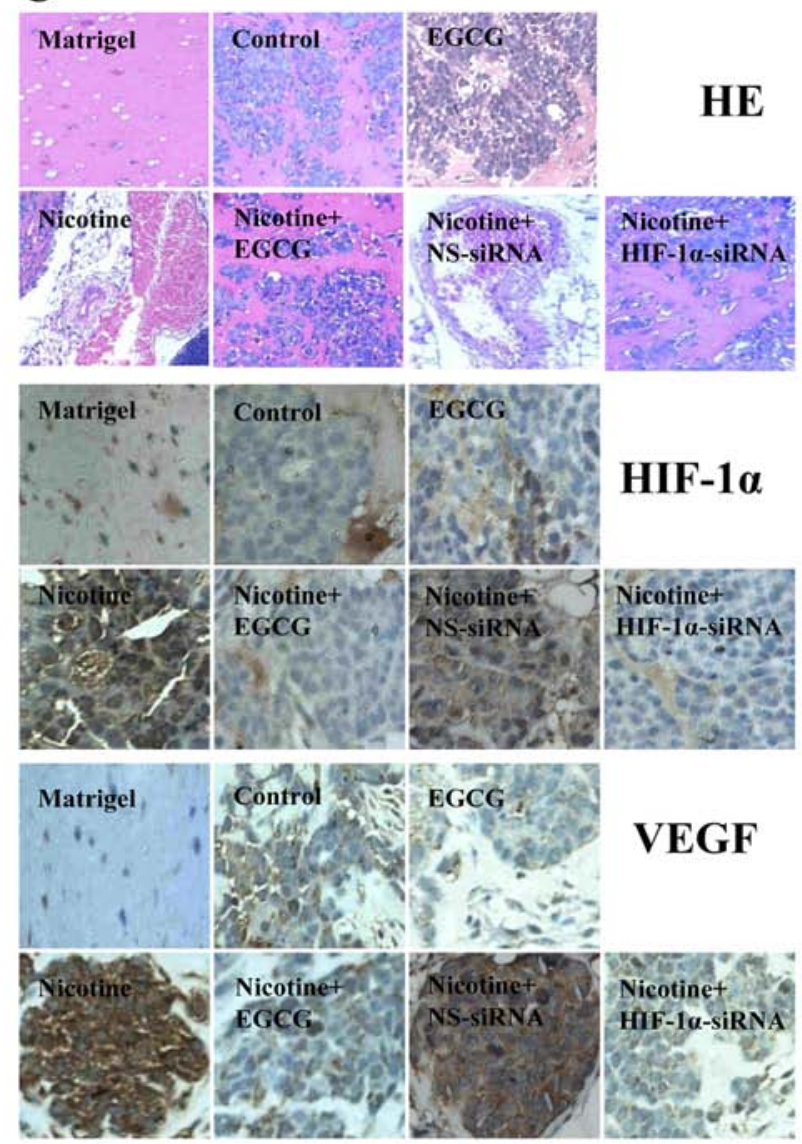

Figure 5. EGCG inhibits nicotine-induced angiogenesis in vivo, and downregulated HIF-1 $\alpha$ and VEGF protein expression in A549 xenografts of nude mice. (A) Representative Matrigel plugs (magnification, x200). (B) Hemoglobin levels in Matrigel plugs. Hemoglobin content was expressed as (mg/g) of Matrigel plug. The data are presented as the mean $\pm \mathrm{SD}$ of three independent experiments. ${ }^{*} \mathrm{P}<0.05$. (C) Immunohistochemical studies on the expression of HIF- $1 \alpha$ and VEGF proteins in A549 xenografted tumors (magnification, x200). The results are representative of three independent experiments. EGCG, epigallocatechin3-gallate; VEGF, vascular endothelial growth factor; HE, hematoxylin and eosin.

the vimentin level is one of the hallmarks of EMT in lung cancer cells (31-33). Therefore, EGCG may be important in suppression of EMT mediated by nicotine in A549 NSCLC cells. Angiogenesis is required for tumor migration, invasion and metastasis (6). In the present study, we found that EGCG also inhibited nicotine-induced angiogenesis in vitro and in vivo. Thus, the results suggest that EGCG-inhibited nicotine-induced migration and invasion may at least occur in part, through the suppression of EMT and angiogenesis.

HIF-1 $\alpha$ and VEGF play an important role in tumor invasion and metastasis, particularly in tumor angiogenesis (34). It has been reported that the expression of HIF-1 $\alpha$ and VEGF was related to a poor prognosis and a worse overall survival (34-36). It has been previously demonstrated that hypoxia-stabilized HIF-1 $\alpha$ promoted EMT through increasing SNAI1 transcription in hepatocellular carcinoma cells (37), and that vascular endothelial growth factor receptor-1 (VEGFR-1) activation enhanced migration and invasion of breast cancer cells through EMT (38). To identify the mechanisms by which EGCG inhibited nicotine-induced angiogenesis and EMT, we determined the expression of HIF-1 $\alpha$ and VEGF. Our results show that EGCG significantly inhibited nicotine-induced HIF-1 $\alpha$ and VEGF protein expression in A549 cells and in A549 xenografts of the nude mice, indicating that EGCG-inhibited nicotine-induced angiogenesis and EMT may be associated with the inhibition of HIF-1 $\alpha$ and VEGF protein expression.

HIF-1 $\alpha$, a key transcriptional factor, governs the expression of a large array of hypoxia-responsive genes, including $V E G F$, $C O X-2$ and $p 53$ (39-42). COX-2, a key regulator of inflammationproducing prostaglandins, can promote tumor cell proliferation and growth. p53, an important tumor suppressor, holds a crosstalk with HIF-1 (42). In the present study, we found that nicotine-induced angiogenesis in vitro and in vivo and VEGF protein expression in A549 cells were HIF-1 $\alpha$-dependent. Additionally, we analyzed the expression of other downstream protein of HIF-1 $\alpha$, including COX-2 and p53. We found that EGCG inhibited nicotine-induced COX-2 protein expression, and concomitantly upregulated the p53 protein level. However, EGCG had no obvious effects on the expression of HIF-1 $\alpha$, VEGF, COX-2 and p53 mRNA mediated by nicotine in A549 cells. These results suggest that EGCG altered the expression of HIF-1 $\alpha$, VEGF, COX-2 and 553 proteins mediated by nicotine through a post-transcriptional mechanism.

The PI3K/Akt and ERK1/2 signaling pathways have been found to be involved in tumor angiogenesis and EMT stimulated by various factors $(43,44)$. These signaling pathways are important in the development and progression of NSCLC $(45,46)$. Therefore, targeting PI3K/AKT and ERK1/2 
signaling pathways may be an emerging treatment strategy for NSCLC $(47,48)$. In the present study, to the best of our knowledge we have for the first time demonstrated that, EGCG suppressed nicotine-induced Akt and ERK1/2 activation, providing the new evidence of a novel molecular mechanism for the anticancer action of EGCG on smoking-associated NSCLC.

In conclusion, to the best of our knowledge, we have demonstrated for the first time that, EGCG significantly inhibited nicotine-induced EMT and angiogenesis in vitro and in vivo, leading to the suppression of migration and invasion in A549 cells. Our findings suggest that EGCG is a potential agent for the prevention and treatment of smoking-associated NSCLC.

\section{Acknowledgements}

We thank Dr Anh D. Le and Dr Qunzhou Zhang (Department of Oral Surgery and Pharmacology, University of Pennsylvania School of Dental Medicine, Philadelphia, USA) for their kind assistance and guidance. The present study was supported by grants from the National Natural Science Foundation of China, nos. 81372511 and 81073103 (to X.T.), the Guangdong Natural Science Foundation S2012010008232 (to X.T.), the Science and Technology of Guangdong Province 2013B031100002 (to X.T.), the Specialized Foundation for Introduced Talents of Guangdong Province Higher Education (Foundation for High-Level Talent) 2050205 (to X.T.), the Zhanjiang Municipal Governmental Specific Financial Fund Allocated for Competitive Scientific and Technological Projects 2012C0303-56 (to X.T.), and the China Scholarship Council and the State Scholarship Fund 201308440331 (to X.T.).

\section{References}

1. Pesch B, Kendzia B, Gustavsson P, Jöckel KH, Johnen G, Pohlabeln H, Olsson A, Ahrens W, Gross IM, Brüske I, et al: Cigarette smoking and lung cancer - relative risk estimates for the major histological types from a pooled analysis of case-control studies. Int J Cancer 131: 1210-1219, 2012.

2. Schaal C and Chellappan SP: Nicotine-mediated cell proliferation and tumor progression in smoking-related cancers. Mol Cancer Res 12: 14-23, 2014.

3. Nair S, Bora-Singhal N, Perumal D and Chellappan S: Nicotinemediated invasion and migration of non-small cell lung carcinoma cells by modulating STMN3 and GSPT1 genes in an ID1-dependent manner. Mol Cancer 13: 173, 2014.

4. Dasgupta P, Rizwani W, Pillai S, Kinkade R, Kovacs M, Rastogi S, Banerjee S, Carless M, Kim E, Coppola D, et al: Nicotine induces cell proliferation, invasion and epithelialmesenchymal transition in a variety of human cancer cell lines. Int J Cancer 124: 36-45, 2009.

5. Wu SQ, Lv YE, Lin BH, Luo LM, Lv SL, Bi AH and Jia YS Silencing of periostin inhibits nicotine-mediated tumor cell growth and epithelial-mesenchymal transition in lung cancer cells. Mol Med Rep 7: 875-880, 2013.

6. Jackson AL, Zhou B and Kim WY: HIF, hypoxia and the role of angiogenesis in non-small cell lung cancer. Expert Opin Ther Targets 14: 1047-1057, 2010.

7. Creighton CJ, Gibbons DL and Kurie JM: The role of epithelialmesenchymal transition programming in invasion and metastasis: A clinical perspective. Cancer Manag Res 5: 187-195, 2013.

8. Zhang Q, Tang X, Zhang ZF, Velikina R, Shi S and Le AD: Nicotine induces hypoxia-inducible factor-1alpha expression in human lung cancer cells via nicotinic acetylcholine receptor-mediated signaling pathways. Clin Cancer Res 13: 4686-4694, 2007.

9. Ma X, Jia Y, Zu S, Li R, Jia Y, Zhao Y, Xiao D, Dang N and Wang Y: $\alpha 5$ nicotinic acetylcholine receptor mediates nicotineinduced HIF-1 $\alpha$ and VEGF expression in non-small cell lung cancer. Toxicol Appl Pharmacol 278: 172-179, 2014.
10. Nurwidya F, Takahashi F, Murakami A and Takahashi K: Epithelial mesenchymal transition in drug resistance and metastasis of lung cancer. Cancer Res Treat 44: 151-156, 2012.

11. Wang L, Zhang X, Liu J, Shen L and Li Z: Tea consumption and lung cancer risk: A meta-analysis of case-control and cohort studies. Nutrition 30: 1122-1127, 2014.

12. Lin IH, Ho ML, Chen HY, Lee HS, Huang CC, Chu YH, Lin SY, Deng YR, He YH, Lien YH, et al: Smoking, green tea consumption, genetic polymorphisms in the insulin-like growth factors and lung cancer risk. PLoS One 7: e30951, 2012.

13. Ravindranath MH, Ramasamy V, Moon S, Ruiz C and Muthugounder S: Differential growth suppression of human melanoma cells by tea (Camellia sinensis) epicatechins (ECG, EGC and EGCG). Evid Based Complement Alternat Med 6: 523-530, 2009.

14. Du GJ, Zhang Z, Wen XD, Yu C, Calway T, Yuan CS and Wang CZ: Epigallocatechin gallate (EGCG) is the most effective cancer chemopreventive polyphenol in green tea. Nutrients 4 : 1679-1691, 2012.

15. Wang F, Chang Z, Fan Q and Wang L: Epigallocatechin-3-gallate inhibits the proliferation and migration of human ovarian carcinoma cells by modulating p38 kinase and matrix metalloproteinase-2. Mol Med Rep 9: 1085-1089, 2014.

16. Zhang W, Yang P, Gao F, Yang J and Yao K: Effects of epigallocatechin gallate on the proliferation and apoptosis of the nasopharyngeal carcinoma cell line CNE2. Exp Ther Med 8: 1783-1788, 2014.

17. Li H, Li Z, Xu YM, Wu Y, Yu KK, Zhang C, Ji YH, Ding G and Chen FX: Epigallocatechin-3-gallate induces apoptosis, inhibits proliferation and decreases invasion of glioma cell. Neurosci Bull 30: 67-73, 2014.

18. Li X, Feng Y,Liu J,Feng X,Zhou K and Tang X: Epigallocatechin3 -gallate inhibits IGF-I-stimulated lung cancer angiogenesis through downregulation of HIF-1 $\alpha$ and VEGF expression. J Nutrigenet Nutrigenomics 6: 169-178, 2013.

19. Zhang Q, Tang X, Lu Q, Zhang Z, Rao J and Le AD: Green tea extract and (-)-epigallocatechin-3-gallate inhibit hypoxia- and serum-induced HIF-1alpha protein accumulation and VEGF expression in human cervical carcinoma and hepatoma cells. Mol Cancer Ther 5: 1227-1238, 2006.

20. He L, Zhang E, Shi J, Li X, Zhou K, Zhang Q, Le AD and Tang X: (-)-Epigallocatechin-3-gallate inhibits human papillomavirus (HPV)-16 oncoprotein-induced angiogenesis in non-small cell lung cancer cells by targeting HIF-1 $\alpha$. Cancer Chemother Pharmacol 71: 713-725, 2013.

21. Liu LC, Tsao TC, Hsu SR, Wang HC, Tsai TC, Kao JY and Way TD: EGCG inhibits transforming growth factor- $\beta$-mediated epithelial-to-mesenchymal transition via the inhibition of Smad2 and Erk1/2 signaling pathways in nonsmall cell lung cancer cells. J Agric Food Chem 60: 9863-9873, 2012.

22. Ko H, So Y, Jeon H, Jeong MH, Choi HK, Ryu SH, Lee SW, Yoon HG and Choi KC: TGF- $\beta 1$-induced epithelial-mesenchymal transition and acetylation of Smad 2 and Smad3 are negatively regulated by EGCG in human A549 lung cancer cells. Cancer Lett 335: 205-213, 2013

23. Takahashi A, Watanabe T, Mondal A, Suzuki K, KurusuKanno M, Li Z, Yamazaki T, Fujiki H and Suganuma M: Mechanism-based inhibition of cancer metastasis with (-)-epigallocatechin gallate. Biochem Biophys Res Commun 443: 1-6, 2014.

24. Nilsson I, Shibuya M and Wennström S: Differential activation of vascular genes by hypoxia in primary endothelial cells. Exp Cell Res 299: 476-485, 2004.

25. Li G, He L, Zhang E, Shi J, Zhang Q, Le AD, Zhou K and Tang X: Overexpression of human papillomavirus (HPV) type 16 oncoproteins promotes angiogenesis via enhancing HIF-1a and VEGF expression in non-small cell lung cancer cells. Cancer Lett 311: 160-170, 2011.

26. Skinner HD, Zheng JZ, Fang J, Agani F and Jiang BH: Vascular endothelial growth factor transcriptional activation is mediated by hypoxia-inducible factor 1alpha, HDM2, and p70S6K1 in response to phosphatidylinositol 3-kinase/AKT signaling. J Biol Chem 279: 45643-45651, 2004

27. Wang L, Gu F, Ma N, Zhang L, Bian JM and Cao HY: CIP2A expression is associated with altered expression of epithelial-mesenchymal transition markers and predictive of poor prognosis in pancreatic ductal adenocarcinoma. Tumour Biol 34: 2309-2313, 2013.

28. Zhao D, Yang K, Tang XF, Lin NN and Liu JY: Expression of integrin-linked kinase in adenoid cystic carcinoma of salivary glands correlates with epithelial-mesenchymal transition markers and tumor progression. Med Oncol 30: 619, 2013. 
29. Balanis N, Wendt MK, Schiemann BJ, Wang Z, Schiemann WP and Carlin CR: Epithelial to mesenchymal transition promotes breast cancer progression via a fibronectin-dependent STAT3 signaling pathway. J Biol Chem 288: 17954-17967, 2013.

30. Xing Y, Qi J, Deng S, Wang C, Zhang L and Chen J: Small interfering RNA targeting ILK inhibits metastasis in human tongue cancer cells through repression of epithelial-to-mesenchymal transition. Exp Cell Res 319: 2058-2072, 2013.

31. López-Novoa JM and Nieto MA: Inflammation and EMT: An alliance towards organ fibrosis and cancer progression. EMBO Mol Med 1: 303-314, 2009.

32. Pirozzi G, Tirino V, Camerlingo R, Franco R, La Rocca A, Liguori E, Martucci N, Paino F, Normanno N and Rocco G: Epithelial to mesenchymal transition by TGF $\beta-1$ induction increases stemness characteristics in primary non small cell lung cancer cell line. PLoS One 6: e21548, 2011.

33. Kim H, Yoo SB, Sun P, Jin Y, Jheon S, Lee CT and Chung JH: Alteration of the E-cadherin/ $\beta$-catenin complex is an independent poor prognostic factor in lung adenocarcinoma. Korean $\mathbf{J}$ Pathol 47: 44-51, 2013.

34. Zhan H, Liang H, Liu X, Deng J, Wang B and Hao X: Expression of Rac1, HIF-1 $\alpha$, and VEGF in gastric carcinoma: Correlation with angiogenesis and prognosis. Onkologie 36: 102-107, 2013.

35. Simonetti O, Lucarini G, Rubini C, Goteri G, Zizzi A, Staibano S Campanati A, Ganzetti G, Di Primio R and Offidani A: Microvessel density and VEGF, HIF-1 $\alpha$ expression in primary oral melanoma: Correlation with prognosis. Oral Dis 19: 620-627, 2013.

36. Kang FW, Gao Y, Que L, Sun J and Wang ZL: Hypoxia-inducible factor- $1 \alpha$ overexpression indicates poor clinical outcomes in tongue squamous cell carcinoma. Exp Ther Med 5: 112-118, 2013.

37. Zhang L, Huang G, Li X, Zhang Y, Jiang Y, Shen J, Liu J, Wang Q, Zhu J, Feng X, et al: Hypoxia induces epithelial-mesenchymal transition via activation of SNAI1 by hypoxia-inducible factor $-1 \alpha$ in hepatocellular carcinoma. BMC Cancer 13: 108-117, 2013.

38. Ning Q, Liu C, Hou L, Meng M, Zhang X, Luo M, Shao S, Zuo X and Zhao X: Vascular endothelial growth factor receptor-1 activation promotes migration and invasion of breast cancer cells through epithelial-mesenchymal transition. PLoS One 8: e65217, 2013.
39. Semenza GL: Defining the role of hypoxia-inducible factor 1 in cancer biology and therapeutics. Oncogene 29: 625-634, 2010.

40. Jiang Y, Cukic B, Adjeroh DA, Skinner HD, Lin J, Shen QJ and Jiang BH: An algorithm for identifying novel targets of transcription factor families: Application to hypoxia-inducible factor 1 targets. Cancer Inform 7: 75-89, 2009.

41. Kaidi A, Qualtrough D, Williams AC and Paraskeva C: Direct transcriptional up-regulation of cyclooxygenase-2 by hypoxiainducible factor (HIF)-1 promotes colorectal tumor cell survival and enhances HIF-1 transcriptional activity during hypoxia. Cancer Res 66: 6683-6691, 2006.

42. Obacz J, Pastorekova S, Vojtesek B and Hrstka R: Cross-talk between HIF and p53 as mediators of molecular responses to physiological and genotoxic stresses. Mol Cancer 12: 93, 2013.

43. Zhang E, Feng X, Liu F, Zhang P, Liang J and Tang X: Roles of PI3K/Akt and c-Jun signaling pathways in human papillomavirus type 16 oncoprotein-induced HIF-1 $\alpha$, VEGF, and IL-8 expression and in vitro angiogenesis in non-small cell lung cancer cells. PLoS One 9: e103440, 2014.

44. Yu H, Zhang L and Liu P: CXCR7 signaling induced epithelialmesenchymal transition by AKT and ERK pathways in epithelial ovarian carcinomas. Tumour Biol: Oct 31, 2014 (Epub ahead of print).

45. Wojtalla A and Arcaro A: Targeting phosphoinositide 3-kinase signalling in lung cancer. Crit Rev Oncol Hematol 80: 278-290, 2011.

46. Zhang J, Cao J, Ma S, et al: Tumor hypoxia enhances non-small cell lung cancer metastasis by selectively promoting macrophage M2 polarization through the activation of ERK signaling. Oncotarget Oct 5: 9664-9677, 2014.

47. Beck JT, Ismail A and Tolomeo C: Targeting the phosphatidylinositol 3-kinase (PI3K)/AKT/mammalian target of rapamycin (mTOR) pathway: An emerging treatment strategy for squamous cell lung carcinoma. Cancer Treat Rev 40: 980-989, 2014.

48. Stinchcombe TE and Johnson GL: MEK inhibition in non-small cell lung cancer. Lung Cancer 86: 121-125, 2014. 